\title{
Review and performance comparison of mechanical-chemical degradation models for lithium-ion batteries
}

\author{
Jorn M. Reniers ${ }^{\mathrm{a}, \mathrm{b}, \mathrm{c}}$, Grietus Mulder ${ }^{\mathrm{b}, \mathrm{c}}$, David A. Howey ${ }^{\mathrm{a}, \mathrm{d}, *}$ \\ ${ }^{a}$ Department of Engineering Science, University of Oxford, OX1 3PJ, Oxford, UK \\ ${ }^{b}$ EnergyVille, Thor Park 8310, 3600, Genk, Belgium \\ ${ }^{c}$ VITO, Boeretang 200, $2400 \mathrm{Mol}$, Belgium \\ ${ }^{d}$ The Faraday Institution, Harwell Campus, OX11 ORA, Didcot, UK
}

\begin{abstract}
The maximum energy that lithium-ion batteries can store decreases as they are used because of various irreversible degradation mechanisms. Many models of degradation have been proposed in the literature, sometimes with a small experimental data set for validation. However, a thorough comparison between different model predictions is lacking, making it difficult to select modelling approaches which can explain the degradation trends actually observed from data. Here various degradation models from literature are implemented within a single particle model framework and their behaviour compared. It is shown that many different models can be fitted to a small experimental data set. The interactions between different models are simulated, showing how some of the models accelerate degradation in other models, altering the overall degradation trend. The effects of operating conditions on the various degradation models is simulated. This identifies which models are enhanced by which operating conditions and might therefore explain specific degradation trends observed in data. Finally, it is shown how a combination of different models is needed to capture different degradation trends observed in a large experimental data set. Vice versa, only a large data set enables to properly select the models which best explain the observed degradation.

keywords: battery, degradation, model, lithium-ion, review
\end{abstract}

\footnotetext{
${ }^{*}$ Corresponding author

Email addresses: jorn.reniers@eng.ox.ac.uk (Jorn M. Reniers), grietus.mulder@energyville.be (Grietus Mulder), david.howey@eng.ox.ac.uk (David A. Howey )

URL: http://epg.eng.ox.ac.uk/howey (David A. Howey )
} 


\section{Introduction}

The amount of energy that a lithium-ion (Li-ion) battery can store decreases over its lifetime. This is the result of various mechanical and electrochemical processes, many of which are influenced by operating conditions. In order to predict the lifetime of a battery the sensitivity of degradation to different load profiles, many models of degradation have been proposed. These can broadly be divided into three categories:

First, empirical models, i.e. parametric functions interpolating a data set from a large scale cycling experiment. These can be very effective, but lack generality because they are only valid for the exact battery chemistry and operating conditions tested in the experiment, and they may exhibit growing inaccuracy of battery health predictions when used for long range extrapolation $[1,2,3,4,5]$.

A second class are physical models. These are typically collections of partial differential equations describing the physical processes taking place in a battery. They are useful for gaining insight into possible degradation mechanisms, and could be more robust than pure empirical approaches, but often are computationally complex, and have many parameters which may be unknown $[3,6,7,8,9]$.

Finally, a more recent development are machine learning models. These are typically black box approaches, similar to the empirical approach, but with greater flexibility. They can be fast and accurate, but require a large data set to be effective, and it is often difficult to interpret and explain their outcomes $[10,11,12,13,14]$.

To investigate the mechanisms of battery ageing, physical models are interesting because they offer a set of testable hypotheses about the underlying reasons for degradation. However, because of the multitude of processes taking place, and the complexity of each, simplifications have to be made, for example by ignoring certain processes, in order to have a computationally tractable approach.

For a single mechanism, i.e. a physical process that causes battery degradation, multiple models, i.e. sets of mathematical equations, have been proposed in the literature. Table 1 gives an overview of the different models that have been proposed. Fig. 1 represents the various models graphically. Most studies only consider a limited number of models describing a few mechanisms. This paper gives a comprehensive overview of the wide range of existing models including specific case studies as examples. They have been implemented here within a flexible modelling approach which allows one to assess their individual effects and interactions. Finally, the impact of variable operating conditions on degradation, according to the different approaches, is investigated, and also, the fitting of a large experimental data set using a combination of models and mechanisms is demonstrated.

The focus of this paper is on lithium-ion chemistries with graphite negative electrodes. An open-access version of the code used to simulate the results in this paper is available on GitHub [15]. 


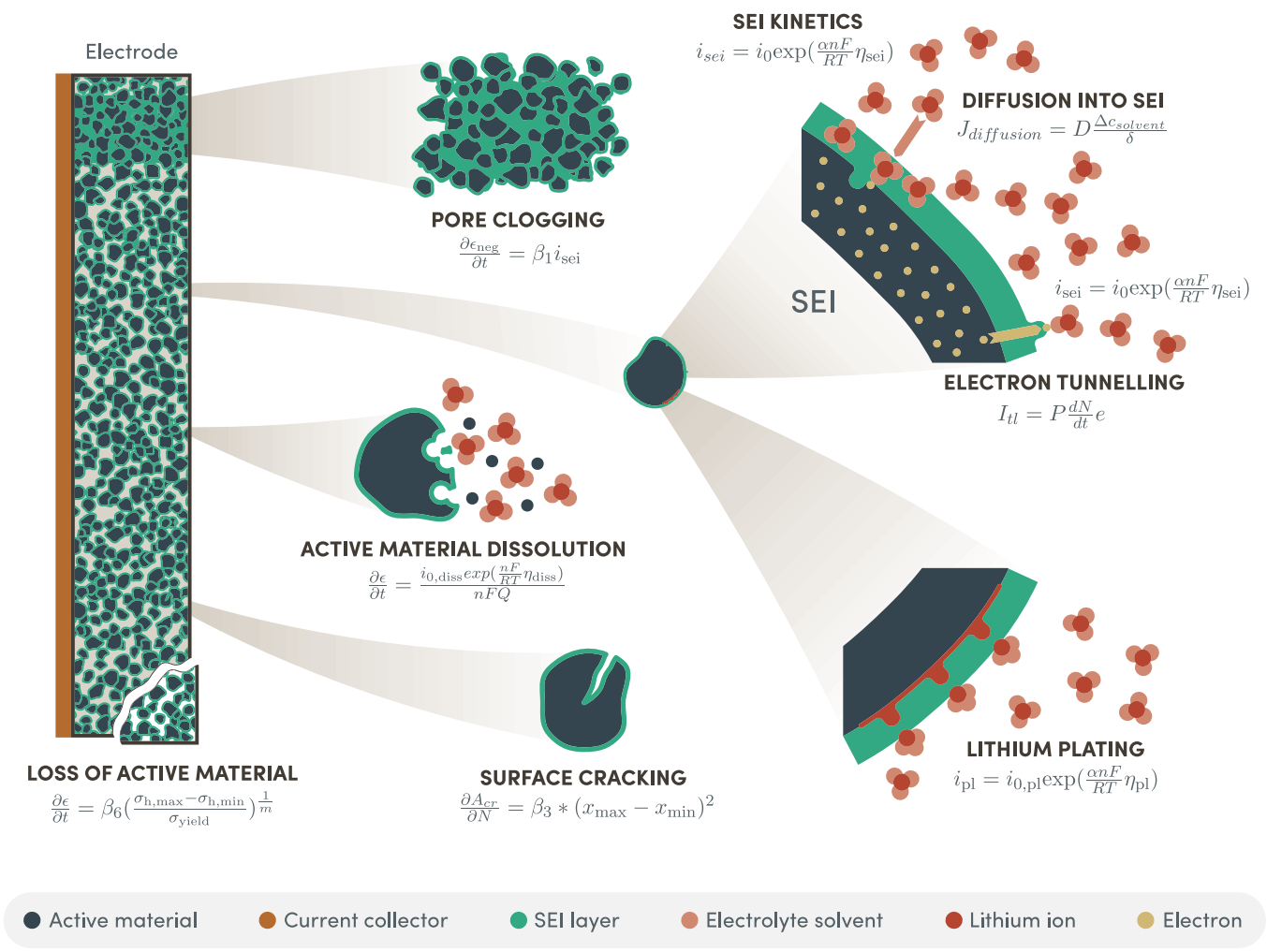

Figure 1: Graphical illustration of the various degradation mechanisms with typical equations modelling each mechanism. 
Table 1: Overview of various degradation models grouped by physical degradation mechanism

\begin{tabular}{|c|c|c|c|c|c|c|c|c|c|c|c|c|c|}
\hline Paper & 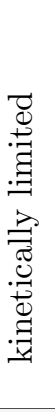 & 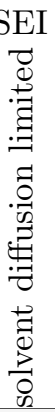 & 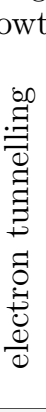 & 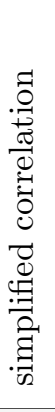 & 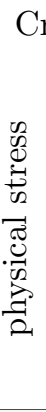 & 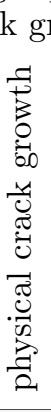 & 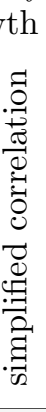 & 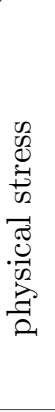 & 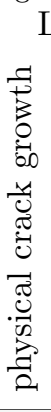 & 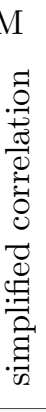 & 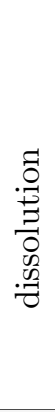 & Li-plating & other \\
\hline Appiah, 2016 [16] & $\mathrm{X}$ & & & & & & & & & & $\mathrm{X}$ & & \\
\hline Ashwin, $2016[17]$ & $\mathrm{X}$ & & & & & & & & & & & & \\
\hline Barai, 2015 [18] & & & & & & & $\mathrm{X}$ & & & & & & \\
\hline Cannarella, 2015 [19] & & & & & & & & & & & & $\mathrm{X}$ & \\
\hline Christensen, 2005 [20] & $\mathrm{X}$ & $\mathrm{X}$ & & & & & & & & & $\mathrm{X}$ & & \\
\hline Delacourt, 2012 [21] & $\mathrm{X}$ & & & & & & & & & $\mathrm{X}$ & & & \\
\hline Deshpande, 2012 [22] & & & & $\mathrm{X}$ & $\mathrm{X}$ & $\mathrm{X}$ & & & & & & & \\
\hline Deshpande, 2017 [23] & & & & $\mathrm{X}$ & & & $\mathrm{X}$ & & & & & & \\
\hline Ekstrom, 2015 [24] & $\mathrm{X}$ & $\mathrm{X}$ & & & & & $\mathrm{X}$ & & & & & & \\
\hline Ge, $2017[25]$ & & & & & & & & & & & & $\mathrm{X}$ & \\
\hline Jin, $2017[26]$ & $\mathrm{X}$ & & & $\mathrm{X}$ & & & & & & $\mathrm{X}$ & & & \\
\hline Kamyab, 2019 [27] & $\mathrm{X}$ & $\mathrm{X}$ & & & & & & & & & & & \\
\hline Kindermann, 2017 [28] & $\mathrm{X}$ & & & & & & $\mathrm{X}$ & & & & $\mathrm{X}$ & & \\
\hline Kupper, 2017 [29] & $\mathrm{X}$ & & & $\mathrm{X}$ & & & & & & & & & \\
\hline Kupper, 2018 [30] & $\mathrm{X}$ & & & $\mathrm{X}$ & $\mathrm{X}$ & & $\mathrm{X}$ & & & & & & $\mathrm{X}$ \\
\hline Laresgoiti, 2015 [31] & & & & & $\mathrm{X}$ & $\mathrm{X}$ & $\mathrm{X}$ & & & & & & \\
\hline Legrand, 2014 [32] & & & & & & & & & & & & $\mathrm{X}$ & \\
\hline Li, $2015[33]$ & & & $\mathrm{X}$ & & & & & & & & & & \\
\hline Lin, $2013[34]$ & $\mathrm{X}$ & & & $\mathrm{X}$ & & & & & & & $\mathrm{X}$ & & $\mathrm{X}$ \\
\hline Narayanrao, 2012 [35] & & & & $\mathrm{X}$ & & & & & $\mathrm{X}$ & $\mathrm{X}$ & & & \\
\hline Ning, $2004[36]$ & $\mathrm{X}$ & & & & & & & & & & & & \\
\hline Pinson, 2013 [37] & $\mathrm{X}$ & $\mathrm{X}$ & & & & & & & & & & & \\
\hline Ploehn, 2004 [38] & & $\mathrm{X}$ & & & & & & & & & & & \\
\hline Purewal, 2014 [39] & & & & $\mathrm{X}$ & $\mathrm{X}$ & $\mathrm{X}$ & & & & & & & \\
\hline Ramadass, 2004 [40] & $\mathrm{X}$ & & & & & & & & & & & & \\
\hline Randall, 2012 [41] & $\mathrm{X}$ & & & & & & & & & & & & \\
\hline Sarafi, 2009 [42] & $\mathrm{X}$ & $\mathrm{X}$ & & & & & & & & & & & \\
\hline Safari, $2010[43]$ & $\mathrm{X}$ & & & & & & $\mathrm{X}$ & & & & & & \\
\hline Safari, 2011 [44] & $\mathrm{X}$ & $\mathrm{X}$ & & & & & & & & & & & \\
\hline Single, 2017 [45] & $\mathrm{X}$ & $\mathrm{X}$ & & & & & & & & & & & \\
\hline Tahmasbi, 2017 [46] & $\mathrm{x}$ & $\mathrm{X}$ & & & & & & & & & & & \\
\hline Tang, $2012[47]$ & $\mathrm{X}$ & & $\mathrm{X}$ & $\mathrm{X}$ & & & & & & & & & \\
\hline Yang, 2017 [48] & $\mathrm{x}$ & $\mathrm{x}$ & & & & & & & & & & $\mathrm{x}$ & \\
\hline
\end{tabular}




\section{Methods}

\subsection{Battery model}

Physical degradation models depend on the underlying physical states, such as lithium concentration at various points in the battery. Therefore, a model is needed in order to calculate these states starting from an initial condition and assuming a given load profile. Various physical battery models exist, depending on the amount of detail they simulate and their computational complexity. One of the simplest such models is the single particle model (SPM), which is relatively fast and can calculate the basic average physical states. However, it can not simulate inhomogeneities and other local effects, for which more complex battery models are needed. Although inhomogeneities can be important for degradation $[49,50,51]$ the SPM was chosen to keep the computational time manageable whilst still capturing the average degradation of an entire cell.

In this study, a negative current means the battery is charging, while a positive current discharges the battery. The terms anode and negative electrode are used interchangeably in this paper, in the usual fashion. Whilst this is correct for discharging, strictly speaking during charging the anode is the positive electrode and the cathode is the negative electrode. The time dependency of most variables has been omitted in the equations for simplicity.

The SPM is a basic 'averaged' electrochemical model of a lithium-ion battery where only solid state diffusion transport and spatially uniform kinetics are accounted for. Each electrode is represented by a sphere. In the sphere, the time-dependent molar lithium concentration $c_{i}(r, t)$ in $\mathrm{mol} / \mathrm{m}^{3}$ is calculated as a function of radius $r$, where subscript $i \in\{-,+\}$ refers to the negative or positive electrode respectively. Fick's law of diffusion relates the time derivative to the gradient and the diffusion constant $D_{i},(1)$,

$$
\frac{\partial c_{i}(r, t)}{\partial t}=\frac{D_{i}}{r^{2}} \frac{\partial}{\partial r}\left(r^{2} \frac{\partial c_{i}(r, t)}{\partial r}\right)
$$

At the centre of the sphere, the gradient has to be zero due to symmetry while at the surfaces, the gradient is equal to the molar flux $j_{i}$, which is related to the current density on that electrode $i_{i}$ via Faraday's constant $F$ and the number of electrons participating in the reaction, $n$. Thus the boundary conditions are

$$
\begin{gathered}
\left.D_{i} \frac{\partial c_{i}(r, t)}{\partial r}\right|_{r=0}=0 \\
\left.D_{i} \frac{\partial c_{i}(r, t)}{\partial r}\right|_{r=R_{i}}=-j_{i}=-\frac{i_{i}}{n F}=-\frac{I}{V_{i} a_{i} n F}
\end{gathered}
$$

The product of the electrode volume $V_{i}$ and the effective electrode surface area $a_{i}$ relates the current density to the total battery current $I$. The specific surface area is a function of the radius of the particle $R_{i}$ 
and the volume fraction of active material $\epsilon_{i}[36]$ according to

$$
a_{i}=3 \frac{\epsilon_{i}}{R_{i}}
$$

The main intercalation reaction happens at the surface of each sphere, and is assumed to follow ButlerVolmer kinetics with a rate constant $k_{i}$, a constant lithium concentration in the electrolyte $c_{e l}$, a transfer coefficient $\alpha$, the maximum lithium concentration $c_{i}^{\max }$, and the ideal gas constant $R$, such that the current density $i_{i}$ can be calculated from the overpotential $\eta_{i}$ at a battery temperature $T$ [36] according to

$$
\begin{gathered}
i_{i}=i_{i, 0}\left(\exp \left(-\frac{\alpha n F}{R T} \eta_{i}\right)-\exp \left(\frac{(1-\alpha) n F}{R T} \eta_{i}\right)\right), \\
\text { where } i_{i, 0}=n F k_{i} c_{i}\left(R_{i}, t\right)^{\alpha} c_{\mathrm{el}}^{1-\alpha}\left(c_{i}^{\max }-c_{i}\left(R_{i}, t\right)\right)^{1-\alpha} .
\end{gathered}
$$

Because temperature is a key determinant of the battery degradation rate, a lumped thermal model was added to the SPM [52]. There are three heat sources, viz. ohmic heating due to the DC resistance of the battery $R_{\text {batt }}$, reaction heating due to the overpotentials, and entropic heating as given by the entropic coefficient of the battery $\frac{\partial O C V}{\partial T}$. Convective heat transfer to the environment at a temperature of $T_{\text {env }}$ with a constant heat transfer coefficient $h$ over a battery surface $A_{\text {batt }}$ cools the battery, which has a heat capacity of $c_{p}$, a density $\rho$, and a cell volume $v$. Thus,

$$
\rho v c_{p} \frac{\partial T}{\partial t}=I^{2} R_{\mathrm{batt}}+I\left(\eta_{\mathrm{neg}}-\eta_{\mathrm{pos}}\right)+I T \frac{\partial O C V}{\partial T}-h A_{\mathrm{batt}}\left(T-T_{\mathrm{env}}\right)
$$

Arrhenius relations with activation energies $E_{D, i}$ and $E_{k, i}$ are used to calculate the temperature dependency of the diffusion and rate parameters starting from the reference values $D_{i}^{\text {ref }}$ and $k_{i}^{\text {ref }}$ at a reference temperature $T^{\text {ref }}$, according to

$$
\begin{aligned}
D_{i} & =D_{i}^{\mathrm{ref}} \exp \left[\frac{E_{D, i}}{R}\left(\frac{1}{T}-\frac{1}{T^{\mathrm{ref}}}\right)\right], \\
k_{i} & =k_{i}^{\mathrm{ref}} \exp \left[\frac{E_{k, i}}{R}\left(\frac{1}{T}-\frac{1}{T^{\mathrm{ref}}}\right)\right] .
\end{aligned}
$$

\subsection{Growth of the SEI layer and loss of lithium}

Many researchers have argued that the most important lithium-ion battery degradation mechanism is the growth of a passivation layer on the graphite electrode. Components of the electrolyte solvent are reduced at 
the graphite surface in a reaction with lithium-ions and electrons from the electrode. The reaction products deposit on the graphite forming the solid electrolyte interphase (SEI) layer [53, 54, 55]. Various reactions have been suggested to occur (depending on the local voltage) $[56,57]$, but the reaction modelled by most researchers is one between ethylene carbonate and lithium ions [29, 36],

$$
2 \mathrm{C}_{3} \mathrm{H}_{4} \mathrm{O}_{3}(l)+2 \mathrm{e}^{-}+2 \mathrm{Li}^{+} \rightleftharpoons\left(\mathrm{CH}_{2} \mathrm{OCO}_{2} \mathrm{Li}\right)_{2}(s)+\mathrm{C}_{2} \mathrm{H}_{4}(g) .
$$

In general, two models have been suggested for this process. Some authors [36, 40] propose a kinetically limited SEI growth model using a Tafel equation, with the exchange current density $i_{0, \text { sei }}$ as a fitting constant. The overpotential for the SEI side reaction $\eta_{\text {sei }}$ is a function of the anode potential $V_{\text {neg }}$, the anode overpotential $\eta_{\text {neg }}$, the equilibrium potential of the SEI growth reaction $V_{\text {sei }}$, and the resistive voltage drop across the existing SEI layer of thickness $\delta$ and specific resistance $r_{\text {sei }}$ :

$$
\begin{gathered}
i_{\text {sei }}=i_{0, \text { sei }} \exp \left(\frac{\alpha n F}{R T} \eta_{\text {sei }}\right), \\
\eta_{\text {sei }}=V_{\text {neg }}+\eta_{\text {neg }}-V_{\text {sei }}+r_{\text {sei }} \delta I .
\end{gathered}
$$

Alternatively, others have suggested a model which includes the limitation caused by diffusion of the electrolyte through the passivation layer $[20,24,27,37,45,46,48]$. By assuming a constant bulk concentration of solvent $c_{\mathrm{s} \text {, bulk }}$ and linear diffusion across the existing SEI layer with a diffusion constant $D_{\text {sei }}$, the concentration at the reaction surface can be calculated. This equation can be substituted into the equation for the exchange current density $i_{0, \text { sei }}=n F k_{\text {sei }} c_{\mathrm{s}}\left(R_{i}, t\right)$, where $k_{\text {sei }}$ is the rate constant and $c_{\mathrm{s}}\left(R_{i}, t\right)$ is the solvent concentration at the particle surface. This results in equation 13 for the SEI side current density, which has one term in the denominator for the reaction kinetics and one for the solvent diffusion. If diffusion is considered to be the rate limiting step, these models predict the typical square root dependency that is often, but not always, seen in the capacity fade over time. If the reaction kinetics is the rate limiting step, the model is very similar to the kinetically limited case. Between those extreme cases, there is a situation where both diffusion and kinetics matter in a similar magnitude.

$$
i_{\mathrm{sei}}=\frac{c_{\mathrm{s}, \mathrm{bulk}}}{\frac{1}{n F k_{\mathrm{sei}} \exp \left(-\frac{\alpha n F}{R T} \eta_{\mathrm{sei}}\right)}+\frac{\delta}{n F D_{\mathrm{sei}}}}
$$

The SEI side reaction and resulting passivation layer growth can have various effects on the battery. First of all, the growth rate of the SEI layer increases linearly with the SEI side current density according to [36] 


$$
\frac{\partial \delta}{\partial t}=\frac{i_{\mathrm{sei}} M}{\rho n F},
$$

where $M$ is the molecular weight of the SEI layer and $\rho$ is its density. Secondly, the side reaction consumes Li-ions which can no longer participate in the main reaction (this is termed 'loss of lithium inventory', or LLI). Therefore, the boundary condition for the lithium diffusion at the surface of the graphite has to be changed to include this side reaction [36]. The lithium concentration gradient at the negative particle surface becomes a function of the main current density on the negative electrode $i_{\text {neg }}$ and SEI side reaction current density $i_{\text {sei }}$ according to

$$
\left.D \frac{\partial c_{\mathrm{neg}}(r, t)}{\partial r}\right|_{r=R}=-\frac{i_{\mathrm{neg}}}{n F}-\frac{i_{\mathrm{sei}}}{n F} .
$$

Thirdly, the SEI layer can block some of the pores of the graphite electrode, resulting in parts of the active material in the electrode no longer being accessible, which increases the current density on the remaining active material. In the single particle model, this can be achieved in an average sense by decreasing the volume fraction of negative active material $\epsilon_{\text {neg, }}$, which will increase the current density according to equations 3 and 4. The equations proposed for pore clogging suggest that the decrease rate of volume fraction of negative active material is a linear function of the SEI side reaction current density [17, 48], with a fitting constant $\beta_{1}$, according to

$$
\frac{\partial \epsilon_{\mathrm{neg}}}{\partial t}=-\beta_{1} i_{\mathrm{sei}} .
$$

Others have argued that the decrease in porosity should affect the diffusion and rate constants also [45], but this is not included here.

\subsection{Surface cracking}

When Li-ions are intercalated, most electrode materials expand, and they subsequently contract on deintercalation. These volume expansion-contraction cycles lead to alternating stresses in the electrodes, which in turn causes crack propagation and material fatigue. When cracks appear at the surface of the electrode, they increase the surface area on which the SEI layer can grow, leading to more loss of cyclable lithium.

To model this, Laresgoiti et al. [31] started with a physical model of the stress and strain in spherical graphite particles and the SEI layer surrounding them, before simplifying it to a correlation between surface concentration and stress. They then used Wöhler curves with slope $m_{1}$ to relate the number of cycles to 
failure to the stress variation during a cycle $\left(\sigma_{\max }-\sigma_{\min }\right)$ relative to the maximum yield strength $\sigma_{\text {yield }}$. Wöhler curves are the result of statistical analysis of metal fatigue [58], and as such the value of $m_{1}$ is determined by fitting the simulation to experimental data. The yield strength is a material property but is treated as a fitting parameter due to a lack of experiments to measure it. Assuming a linear damage accumulation, Laresgoiti et al. related the lost charge capacity $Q_{\text {lost }}$ per cycle $N$ to this mean stress with a fitting parameter $\beta_{2}$, according to

$$
\frac{Q_{\text {lost }}}{N}=\beta_{2}\left(\frac{\sigma_{\max }-\sigma_{\min }}{\sigma_{\text {yield }}}\right)^{\frac{1}{m_{1}}} .
$$

Alternatively, Deshpande et al. [23] started from a physical stress model, which they then simplified to a quadratic relation between the increase in crack surface area $A_{\mathrm{cr}}$ and the concentration swing over that cycle, with fitting parameter $\beta_{3}$. The concentration swing is the difference between the highest and lowest Li-fractions $x$ during the cycle. The Li-fraction is the lithium concentration relative to the maximum lithium concentration $x=c_{i} / c_{i}^{\max }$. Thus,

$$
\frac{\partial A_{\mathrm{cr}}}{\partial N}=\beta_{3}\left(x_{\max }-x_{\min }\right)^{2}
$$

Others follow a more empirical approach. Barai et al. [18] assume that crack growth increases exponentially with charge throughput until it plateaus at a (constant) maximum crack surface area $A_{\text {max }}$ This means that the time derivative of the crack surface area is proportional to the existing crack surface area and the absolute value of the current with a fitting constant $\beta_{4}$. They argue that these cracks also increase the tortuosity, resulting in a decreasing effective diffusion constant $D_{\text {neg }}^{\text {eff }}$ with a fitting parameter $\beta_{5}$. This can be expressed as

$$
\begin{gathered}
\frac{\partial A_{\mathrm{cr}}}{\partial t}=\beta_{4}\left(A_{\max }-A_{\mathrm{cr}}\right)|I|, \\
D_{\mathrm{neg}}^{\mathrm{eff}}=D_{\mathrm{neg}}\left(1-\frac{A_{\mathrm{cr}}}{A_{\max }}\right)^{\beta_{5}} .
\end{gathered}
$$

Ekstrom et al. and Kindermann et al. [24, 28] assumed that additional SEI growth due to crack growth could be simulated by a second side reaction current which follows a Tafel equation. The difference compared to the original SEI side reaction is that the rate constant of the crack growth reaction $k_{\mathrm{cr}}$ is a function of the Li-fraction $x_{n e g}$, to represent the phase transitions in the graphite. Thus the side reaction current is

$$
i_{\mathrm{cr}}=n F k_{\mathrm{cr}}\left(x_{\mathrm{neg}}\right) \exp \left(\frac{\alpha n F}{R T} \eta_{\mathrm{sei}}\right) .
$$




\subsection{Loss of active material}

The previous section was about cracks initiating at electrode surfaces and growing. Similar underlying physical phenomena (such as alternating stresses) can also lead to cracks forming within electrodes. This can cause loss of electrical contact, and a reduction of the usable active material.

Several researchers have developed mechanical stress models for spherical particles of radius $R_{0}[59,60$, $61,62,63]$. They all arrive at similar equations for the radial stress $\sigma_{r}$ and the tangential stress $\sigma_{t}$ at radius $\mathrm{r}$, where $\Omega$ is the partial molar volume, $E$ is the Young's modulus, $\nu$ is the Poisson's ratio, $c$ is the Li-concentration as a function of the radius and $\zeta$ is a dummy integration variable. The equations for radial and tangential stress, respectively, are

$$
\begin{gathered}
\sigma_{r}(r)=\frac{2 \Omega E}{3(1-\nu)}\left(\frac{1}{R_{0}^{3}} \int_{0}^{R_{0}} c(r) r^{2} \mathrm{~d} r-\frac{1}{r^{3}} \int_{0}^{r} c(\zeta) \zeta^{2} \mathrm{~d} \zeta\right), \\
\sigma_{t}(r)=\frac{\Omega E}{3(1-\nu)}\left(\frac{2}{R_{0}^{3}} \int_{0}^{R_{0}} c(r) r^{2} \mathrm{~d} r+\frac{1}{r^{3}} \int_{0}^{r} c(\zeta) \zeta^{2} \mathrm{~d} \zeta-c\right) .
\end{gathered}
$$

The radial and tangential stress can be combined into the hydrostatic stress $\sigma_{h}$, given by

$$
\sigma_{h}(r)=\frac{\sigma_{r}(r)+2 \sigma_{t}(r)}{3}
$$

These authors did not link this stress to a degradation effect, but the previous section provided many correlations between stress and crack growth. Because the underlying principles are the same, these correlations can also be used to calculate the loss of active material (LAM) in the electrodes. The LAM can be simulated by decreasing the volume fraction of active material $\epsilon$ according to

$$
\frac{\partial \epsilon}{\partial t}=\beta_{6}\left(\frac{\sigma_{\mathrm{h}, \max }-\sigma_{\mathrm{h}, \min }}{\sigma_{\text {yield }}}\right)^{\frac{1}{m_{2}}}
$$

which is similar to equation 17 . This will increase the current density on the remaining active material according to equations 3 and 4 and therefore decrease the capacity of the cell. As before, the parameters $\beta_{6}$, $m_{2}$, and $\sigma_{\text {yield }}$ are fitting parameters.

Delacourt and Safari [21] made an empirical model where the volume fraction decreases as a function of the current density $i$ according to

$$
\frac{\partial \epsilon}{\partial t}=\beta_{7}(T)|i|+\beta_{8}(T) \sqrt{|i|}
$$

with fitting parameters $\beta_{7}$ and $\beta_{8}$, which are temperature dependent. Jin et al. [26] used a similar 
equation but with $\beta_{8}=0$.

Narayanrao et al. [35] modelled LAM by decreasing the specific surface area $a$ directly rather than decreasing the volume fraction $\epsilon$, which indirectly achieves the same effect, namely increasing the current density on the remaining active material as given by equations 3 and 4 . They assumed that the effective surface area decreases proportionally to itself with rate constant $\beta_{9}$, according to

$$
\frac{\partial a}{\partial t}=-\beta_{9} a
$$

Kindermann et al. [28] modelled cathode dissolution rather than stress-based loss of active material. They assumed this process is inversely proportional to the maximum Li-concentration per unit of surface area $q=c_{i}^{\max } l_{i}$ where $l_{i}$ is the electrode thickness. They further assume dissolution has Tafel kinetics with a constant exchange current density $i_{0, \text { diss }}$ and overpotential $\eta_{\text {diss }}$, which is calculated similarly to the overpotential for the SEI side reaction given by equation 12 . The resulting equation is

$$
\frac{\partial \epsilon_{\mathrm{pos}}}{\partial t}=\frac{i_{0, \mathrm{diss}} \exp \left(\frac{n F}{R T} \eta_{\mathrm{diss}}\right)}{n F q}
$$

\subsection{Lithium plating}

Li-ions can be deposited as metallic lithium instead of intercalating in the electrodes, leading to loss of cyclable lithium and of capacity, as well as possible safety issues. Most researchers suggest that this process follows standard Butler-Volmer or Tafel kinetcs [19, 25, 32, 48]. The degradation effects of the plating side reaction current $i_{\mathrm{pl}}$ are exactly the same as for the SEI growth side reaction in terms of growing a layer and clogging the anode pores. It has been shown that the plated lithium can be partially re-inserted in the electrode, thus recovering the lost capacity [64], but this is not included in the model. The equations used to describe lithium plating are very similar to the kinetically limited SEI growth model, namely

$$
\begin{gathered}
i_{\mathrm{pl}}=i_{0, \mathrm{pl}} \exp \left(\frac{\alpha n F}{R T} \eta_{\mathrm{pl}}\right), \\
\eta_{\mathrm{pl}}=V_{\text {neg }}+\eta_{\text {neg }}-V_{\mathrm{pl}}+r_{\mathrm{sei}} \delta I .
\end{gathered}
$$

\subsection{Other degradation mechanisms}

Various other degradation mechanisms exist, such as electrolyte drying, gas formation, current collector corrosion, etc. [65]. They are not implemented in this model at this stage due to lack of agreement in the literature regarding their implementation. 


\subsection{Model implementation and solution}

The diffusion equation was discretised in space using Chebyshev collocation [66]. The battery and degradation models were then formulated as a state space model, with the load current as input and the outputs as the derivatives of all the states. Forward Euler time integration was used to solve this equation system over time.

A challenge with implementing some of these degradation models is that they depend on cycle count rather than time. In degradation experiments with well defined cycles, this might be possible. But when real-life battery usage is predicted, it is very difficult to define what a single cycle means. Some researchers have used rainflow counting to solve this problem $[1,67]$, but so far experimental proof that this is justified is lacking. For the purpose of this work, it was assumed that there is a linear relationship between the cycle count and calendar time in order to make the results more general without deviating too much from the original models.

Similarly, some other models had to be adapted to fit in the framework of this work. The Li-fraction evolves monotonically during a half cycle so equation 18 can be approximated by using the difference in Li-fraction between the previous and present time steps, which will give the difference between minimum and maximum values over the entire cycle. A similar approach was followed for equation 17, where it was additionally assumed that the effect was to increase the crack surface area $A_{\mathrm{cr}}$ rather than directly decreasing the capacity. The full implementation of the code is available on GitHub [15].

For the rest of this paper, capacity is defined as the charge accessible in a cell between the allowed voltage limits. In the experiments, it is measured by integrating the current while charging the cell from its lower voltage limit to the maximum voltage with a CC CV current. In the simulations, the same CC CV charge between the same voltage limits is simulated and the simulated capacity is then the integral of the current during this charge. Both in the experiments and simulations, a such capacity check is done after a predefined number of cycles.

\section{Results and discussion}

\subsection{Degradation data and parameter fitting}

The simulations according to the various models are now compared with experimental data from two cells. A small number of degradation experiments had been conducted by various partners in the Everlasting project [68] using a high-energy LG Chem NMC 18650 cell (INR18650 MJ1, [69]). This cell was cycled with a constant current, both on charge and on discharge, at $25^{\circ} \mathrm{C}$. This was done between 10 and $90 \%$ state of charge (SoC). The data used in this paper is from a cell which was charged at $1 \mathrm{C}$ and discharged at $1.5 \mathrm{C}$. 
A larger degradation experiment had been conducted by various partners in the Mat4Bat project [70] using a high power Kokam NMC prismatic cell (SLPB78205130H, [71]). These cells were cycled with a constant current and constant voltage charge, and a constant current discharge. This was at various temperatures, and between various $\mathrm{SoC}$ windows [3].

The parameters of the SPM were fitted twice, once for each cell type. This was done manually by comparing the simulated and measured voltage during charging and discharging at various currents. The fitting parameters of the degradation models were set differently for every result shown below in order to match the simulations with the data. For example, when only SEI growth is considered, the values of the diffusion constant $D_{\mathrm{SEI}}$ and the exchange current density $i_{0 \text {,sei }}$ were set such that the predicted degradation matched the data. When later SEI growth was combined with other degradation mechanisms, such as surface cracking, the diffusion constant and exchange current density were changed such that the total predicted degradation fitted the data.

\subsection{Classification of degradation models}

Three basic trends can be observed within existing lithium-ion battery degradation data sets. Some types of cells degrade faster at the start of life and their degradation rate decreases later in life [72, 73]. The degradation behaviour of other cells is more linear with time, with rate of capacity decrease being approximately constant for a fixed usage pattern $[74,75]$. Other cells show an accelerating degradation, especially towards the end of their lifetime when the capacity suddenly decreases very strongly [48, 76, 77]. Sometimes, different operating conditions can lead to different degradation trends for the same cell type.

The degradation model equations previously introduced determine the trend of degradation over time and with usage. The kinetically limited SEI growth model (equation 11) will produce a constant capacity fade for fixed usage because the magnitude of the side reaction current is broadly independent of the past degradation. On the other hand, the diffusion limited SEI growth model (equation 13) will predict a decreasing rate of degradation for fixed usage because the magnitude of the side reaction current is inversely proportional to past degradation, represented by the thickness of the existing SEI layer $\delta$, which will produce a square root of time dependency.

Laresgoiti's model for surface cracking (equation 17) will give a constant degradation trend because the stress is not affected by previous crack growth. The same applies for Deshpande's model (equation 18). Barai's crack growth model (equation 19) will give a decreasing trend because the bigger the crack surface, the smaller the remaining possible active area for crack growth $A_{\max }-A_{\mathrm{cr}}$. Ekstrom's model (equation 21) will result in a constant degradation trend for the same reason as the kinetically limited SEI growth model.

There is more variability in the effects of the models for loss of active material. The physical stress model 
from Dai et al. coupled with a proportional degradation model from Laresgoiti et al. (equations 22 to 25) will give a strongly accelerating degradation trend with time, because the more active material is lost, the higher the current density, the higher the Li-concentration gradients, the higher the stress, and the higher the decrease in porosity. For similar reasons, Delacourt's model (equation 26) will also give an increasing trend but according to a square root. On the other hand, Narayanrao's loss of active material model (equation 27) will give an exponentially decreasing trend because the smaller the effective surface $a$, the less it will decrease. Kindermann's model for electrode dissolution (equation 28) will give a constant degradation rate, just like kinetically limited SEI growth. Finally, the Li-plating model (equation 29) predicts a more or less constant degradation rate, because the rate of lithium plating does not depend on the previous amount of plated lithium.

As an example, Fig. 2 shows the different models classified by the trends they predict, along with a data set for each trend. None of the surface crack growth models are included because surface cracks don't decrease the capacity on their own. They only enlarge the surface area, and it is the SEI growth on this additional surface which will cause extra degradation. Therefore, crack growth models only cause extra degradation when they are combined with a model for SEI growth. It should be noted that the different trends can be difficult to see in the simulation results of Fig. 2 because in some cases the rate of change of the gradient is very gradual within the window shown. For instance, the capacity loss according to an exponential process is more or less linear at the start of the battery life (since $y=e^{-t / \tau} \approx 1-t / \tau$ for large $\tau$ and small $t$ ). Some exponential processes can decrease the capacity very suddenly, as is the case for Delacourt's LAM model. At the last successful capacity check in the simulation, the cell still had about $70 \%$ remaining capacity but then the cell degraded very quickly until it had no more remaining capacity.

\subsection{Combined models}

Fig. 3 shows an example set of results when an SEI growth model is combined with one other degradation model. As explained in the section on degradation data and parameter fitting, the parameters for the degradation models were set differently for every combination of models in order to give a best fit against the available data. For reference, the solid lines show the degradation according to the SEI model alone; each different colour represents a different value of the diffusion constant and/or exchange current density in the model. The dashed and dotted lines give combinations of SEI models with one other model, as indicated, such that the difference between the dashed/dotted and solid lines represents the additional degradation due to the second degradation model. The data and load cycles are the same as for Fig. 3.

The combination of a diffusion limited SEI growth model (which results in decelerating degradation over time) with another decelerating or linear degradation model produces a decreasing trend over time. Combin- 

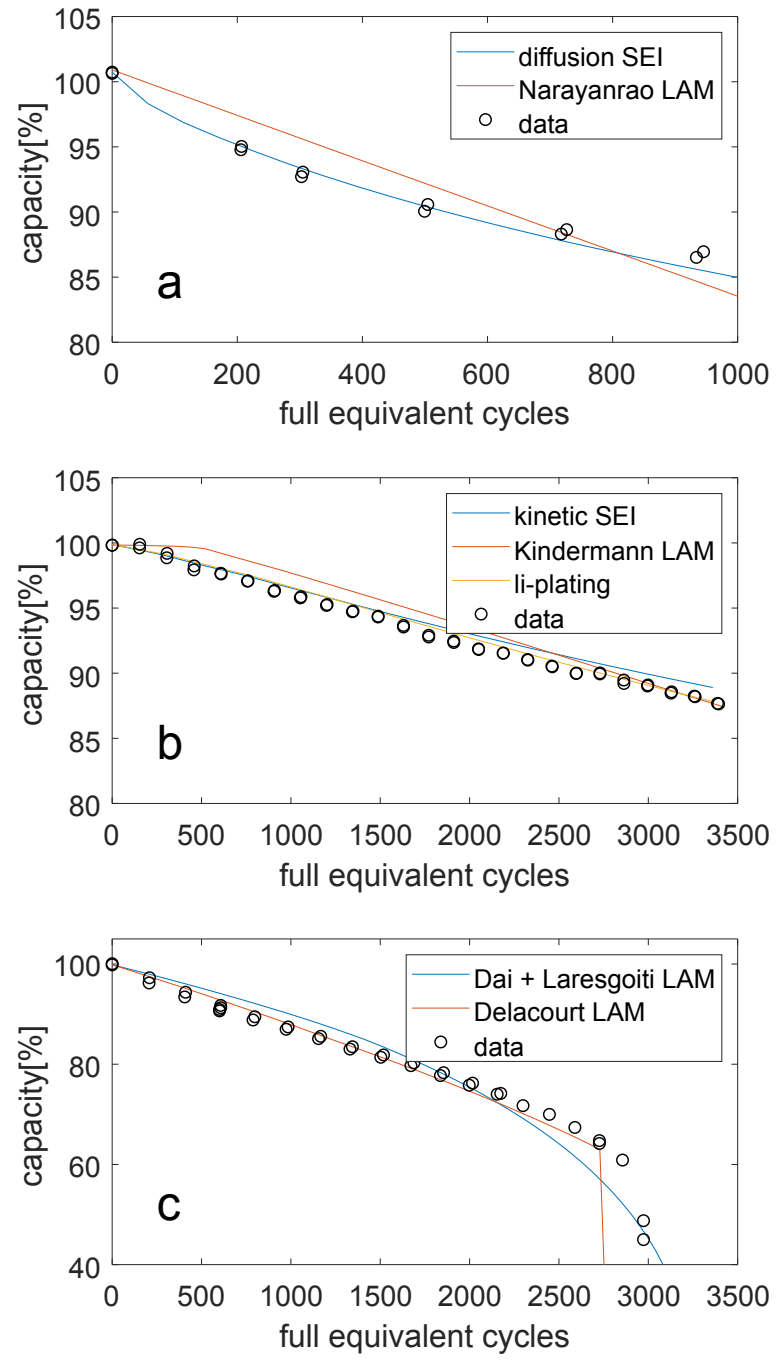

Figure 2: Basic degradation trends of the individual degradation models. The black markers are experimental results as described in the section about degradation data, while the lines are the simulations according to the various models. (a) Decreasing degradation rate for the LG Chem cell; (b) constant degradation rate for the Kokam cell cycled at $25^{\circ} \mathrm{C}$ at a $1 \mathrm{C}$ charge and $1 \mathrm{C}$ discharge between 10 and $90 \% \mathrm{SoC}$; (c) increasing degradation rate the Kokam cell cycled at $45^{\circ} \mathrm{C}$ at a $3 \mathrm{C}$ charge and $1 \mathrm{C}$ discharge between 0 and $100 \%$ SoC. 

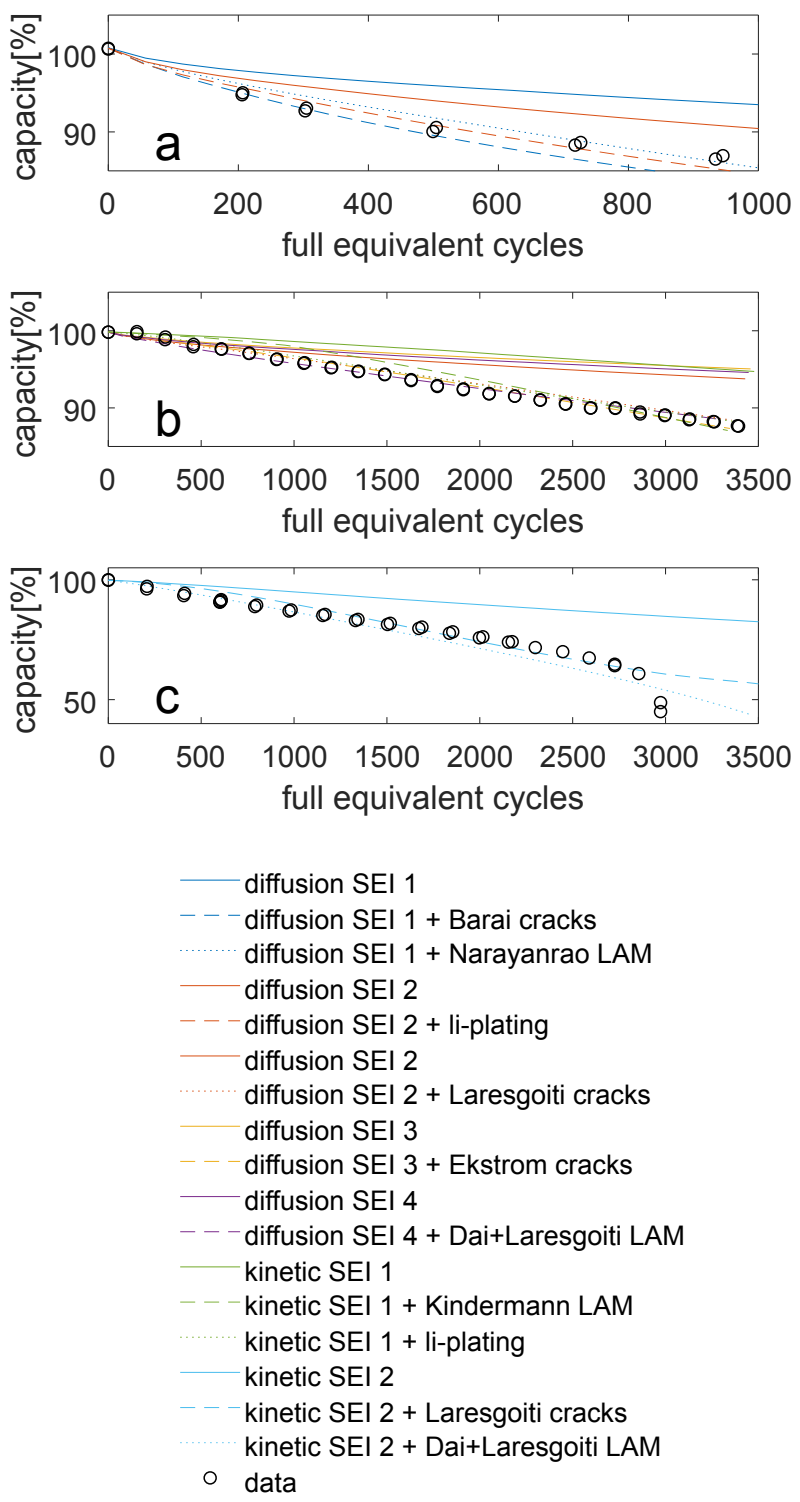

Figure 3: The dashed and dotted lines show the results of combining an SEI model with one other degradation model. For reference, the solid line of the same colour indicates the degradation according to the SEI model only. The different colours represent SEI models with different diffusion constants and/or exchange current densities. The black data points are the same as in Fig. 2. (a) Decreasing degradation rate; (b) Constant degradation rate; (c) Increasing degradation rate. 
ing a diffusion limited SEI model with an accelerating degradation model produces a constant degradation rate if the rates of acceleration and deceleration are similar. The same effect also results from combining two models each having a constant degradation rate. Adding an accelerating degradation model to a kinetically limited SEI growth model produces an accelerating trend. The crack growth models are a slight exception: the SEI layer grows on the total electrode surface area, implying that the degradation rate is proportional to the integral of the crack growth rate. Therefore, a crack growth model with a constant crack growth rate will give an increasing degradation trend.

When implementing the combination of two models, it is important to include the feedback mechanisms between them. For instance, if the active material of the graphite electrode decreases due to a LAM degradation model, the current density for the same applied current will increase. This will lead to higher overpotentials, which will enhance the SEI growth if a kinetically limited SEI growth model is used. This effect is shown on Fig. 4, with Fig. 4(a) indicating the SEI layer thickness and Fig. 4(b) indicating the electrode surface area. The blue area shows the model results when only the SEI growth is modelled; as can be seen the electrode surface area does not change in this scenario, and the layer thickness grows almost linearly with cycling assuming it is only kinetically limited. The yellow area shows the increase in the SEI layer growth and surface area respectively when LAM is modelled alongside SEI growth. Although the LAM model alone produces a constant degradation rate, when combined with the SEI growth model it results in an accelerating growth rate. This is for the same reason as for crack growth described in the previous paragraph: the SEI growth rate is proportional to the total active material, which is linearly increasing, not to the rate at which this material is lost, which is constant. Note that this feedback mechanism does not exist when a purely diffusion limited SEI growth model is used because in that case, higher overpotentials do not lead to higher SEI growth rates. There are other similar cases, e.g. when the diffusion constant is reduced due to crack growth as given by equation 20 and active material is lost due to a physical stress model which is given by equations 22 to 25 .

Negative feedback also exists, for example between kinetically limited SEI growth and Li-plating. Both models remove cyclable lithium, which increases the anode potential, while both are enhanced by lower voltages with respect to $\mathrm{Li}$. This means that if there is more/less SEI growth, the anode potential is higher/lower and there will be less/more Li-plating.

Finally, either electrode can be limiting. For example, Kindermann's degradation model, given by equation 28 , acts on the cathode, while the SEI growth models decrease the anode capacity by removing Li-ions irreversibly. If the SEI model is dominant, the capacity is limited by the anode, and adding Kindermann's degradation model will have no effect on the overall capacity and vice versa. 

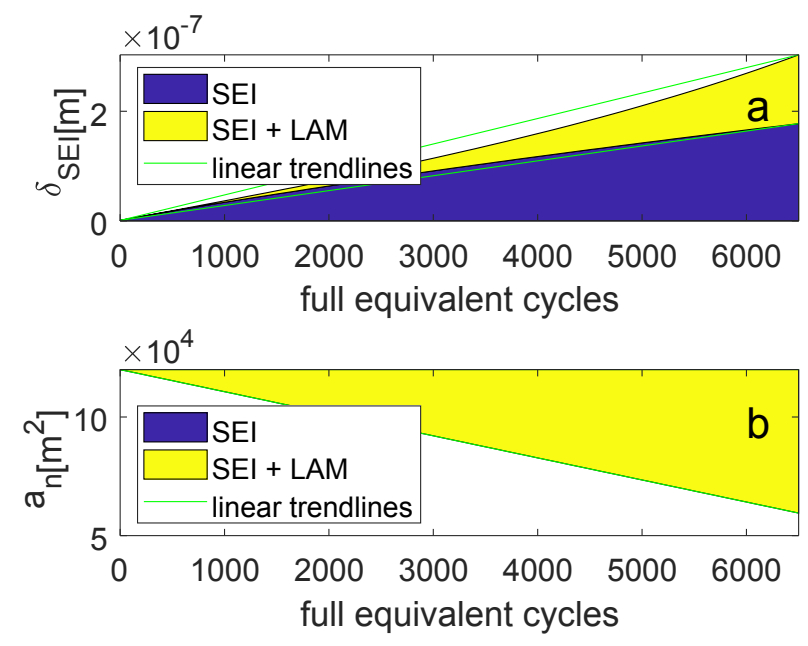

Figure 4: Feedback between kinetically limited SEI growth and LAM. The blue area is the effect when only SEI growth is considered, while the yellow area gives the additional effect when both SEI growth and LAM are considered. (a) Thickness of the SEI layer; (b) Effective surface area.

\subsection{Dependence of degradation on usage}

It is well known that battery degradation is influenced by operational usage factors such as current, SoC and temperature $[1,4]$. The different models of degradation respond very differently to varying operating conditions, as shown in Fig. 5.

The kinetically limited SEI growth model predicts increased degradation at high current, high SoC and high temperature, especially if the temperature dependency of the rate constant is explicitly considered, similar to equation 9. The diffusion limited SEI growth model is however independent of current and SoC and will only give higher degradation at higher temperature, again on the condition that the temperature dependency of the diffusion constant is considered, similar to equation 8. Therefore, in the case of diffusion limited SEI growth, the degradation of all cycles with the same temperatures maps onto a single line if plotted against time on the x-axis. However, the x-axis of Fig. 5 is 'full equivalent cycles' (FEC), which is the total charge throughput divided by twice the nominal cell capacity. Because the different cycles take different amounts of time, the prediction does not map to a single line per temperature. This shows how changing the independent variable can reveal different trends in degradation data, which might be useful to identify which degradation models fit the data best.

There is a large difference between the results of the various models for crack growth as well as between the various models for LAM. The most important difference is how much the stress is affected by they change of the diffusion constant due to changing temperatures. Lower temperatures and corresponding lower diffusion constants will always lead to larger spatial concentration gradients inside the active material, as well as to faster concentration swings on the surface of the electrode. Desphande's crack growth model and Dai's LAM 

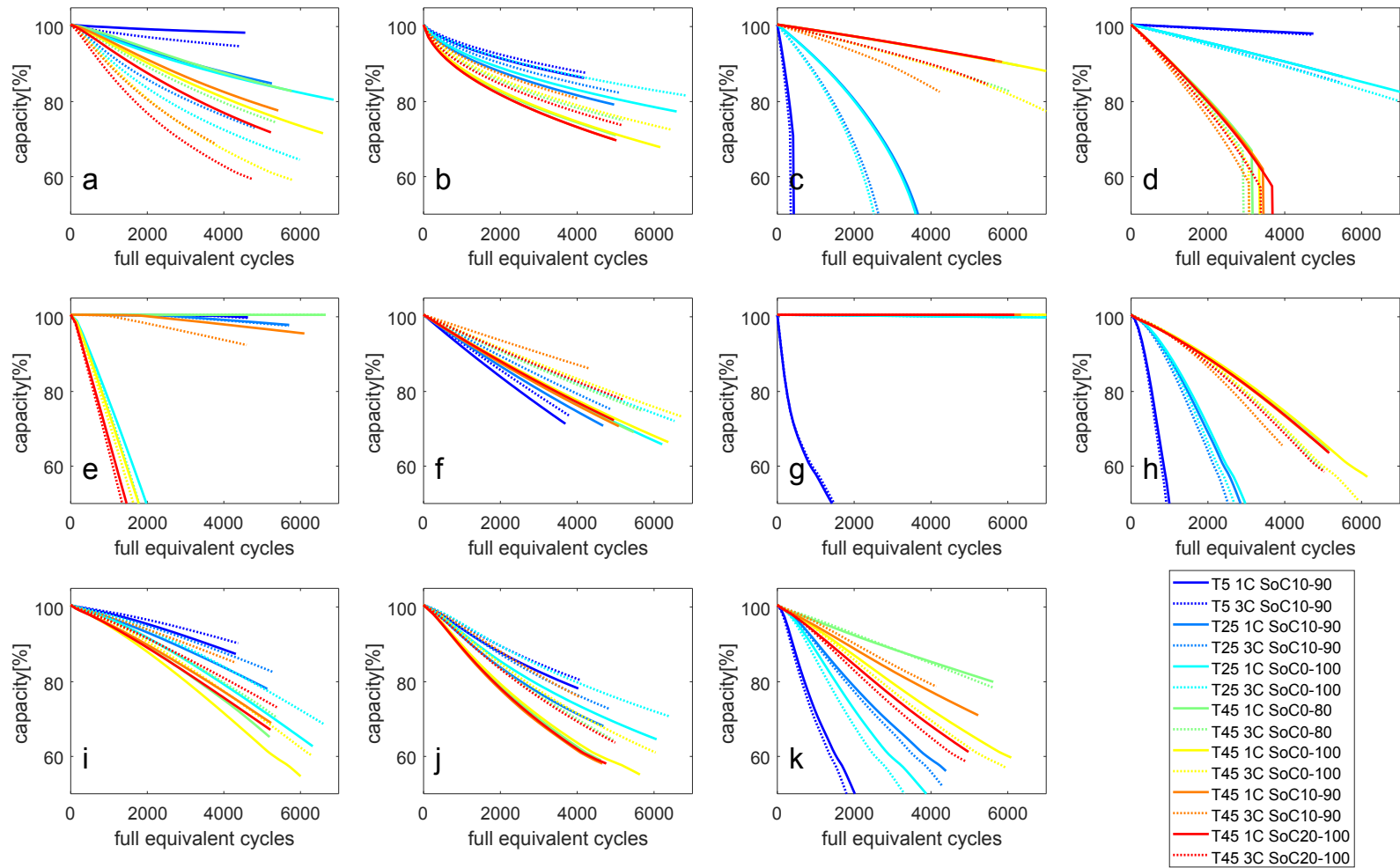

Figure 5: Dependencies of the various degradation models on usage. Each subplot gives the predictions according to one model, with the exception of the crack growth models which are combined with a diffusion limited SEI growth model. Discharge is at $1 \mathrm{C}$, and charging is CC CV at various currents indicated by the legend. Ambient temperatures and SoC windows are indicated in the legend too. (a) Kinetically limited SEI growth, equation 11; (b) Diffusion limited SEI growth, equation 13; (c) Dai + Laresgoiti LAM, equations 22 to 25; (d) Delacourt LAM, equation 26; (e) Kindermann LAM, equation 28; (f) Narayanrao LAM, equation 27; (g) Li-plating, equation 29; (h) Diffusion limited SEI growth + Deshpande crack growth, 18; (i) Diffusion limited SEI growth + Laresgoiti crack growth, equation 17; (j) Diffusion limited SEI growth + Barai crack growth, 19; (k) Diffusion limited SEI growth + Ekstrom crack growth, equation 21.

model, respectively equations 18 and 22 to 25, are more sensitive to this than Laresgoiti's crack growth model (equation 17). Other models are independent of any operating condition, such as Narayanrao's LAM model (equation 27). Such models will predict identical degradation for all cycles of the same temperature, but because the independent variable is FEC instead of time, the degradation for each cycle looks different. The more empirical models such as equations 19 and 26 typically only depend on the current and temperature, and given that the independent variable is proportional to the charge throughput, this means all predictions for the same temperature map almost to a single line. The models using Tafel equations (equations 21, 28 and 29) have the same dependencies as the kinetically limited SEI growth model although the temperature dependency of equations 21 and 29 is negative instead of positive.

Fig. 6 demonstrates how a combination of various models can generalise to match most of the trends observable in the large data set recorded for the Kokam cell. The generalised model illustrated here is a combination of diffusion limited SEI (equation 13), Delacourt's LAM model (equation 26), Kindermann's model for cathode dissolution (equation 28) and Yang's model for Li-plating (equation 29). All the models 


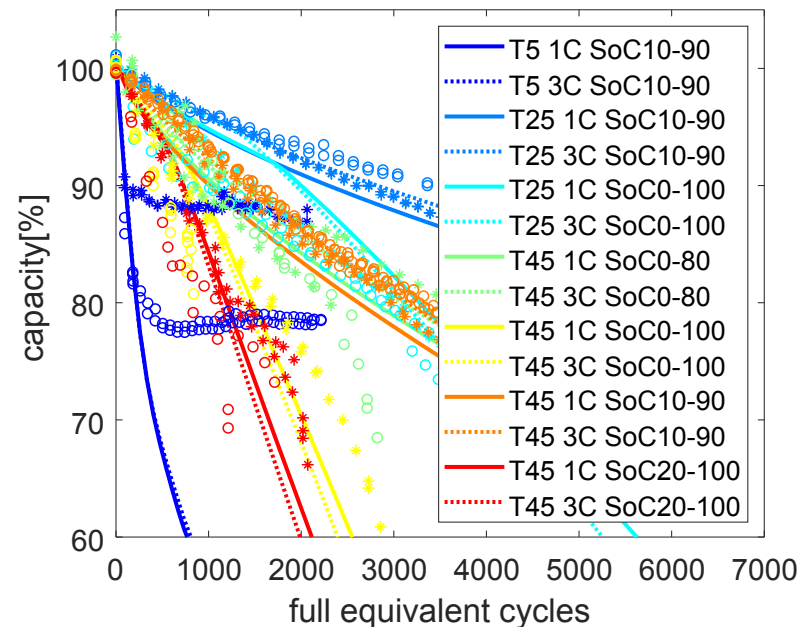

Figure 6: Degradation predictions for various cycling regimes, comparing a generalised model (lines) vs. measured degradation data from Kokam cells with the same cycling regimes as in Fig. 5 (circles for 3C data, asterisks for 1C data). The generalised model combines a diffusion limited SEI model, Delacourt's LAM model, Kindermann's model for cathode dissolution and Yang's model of Li-plating.

interact with each other as explained previously. A best fit of this generalised model is shown across the entire experimental data set. The Li-plating model ensures the rapid degradation at low temperature is captured, but fails to capture the later decreasing degradation trend fast enough. It is unclear why this happens, but probably some negative feedback mechanism prevents further plating in the real cell, which is not included in the model. The cathode dissolution model explains the degradation for cycles at high SoC windows, and the combination of the SEI layer growth model and loss of active material model explains the remaining degradation trends.

\section{Conclusions}

In this work, many different physical degradation models from literature were implemented within a single particle model framework. When looking at the partial differential equations of the models, it is straightforward to identify the basic degradation trend that each will predict: a decreasing, constant, or increasing degradation rate.

Combining two degradation models might result in a different degradation trend. For instance the combination of one model with decreasing and one with increasing degradation trends can result in a constant degradation rate. However, feedback mechanisms between the models might alter the trends that would normally be expected. For instance, the positive feedback between a kinetically limited SEI growth model and a LAM model, both of which have a constant degradation trends, results in an increasing trend. Also negative feedback mechanisms exist, for instance between lithium plating and kinetically limited SEI growth.

When considering a small degradation data set, it might seem as if all the models behave similarly 
and all can predict the observed degradation. But when variable cycling conditions are simulated, the differences between the models become apparent. Fitting a large data set requires multiple degradation models to capture the different degradation trends observed for different operating conditions. Therefore, when deciding which degradation model to choose to explain a given data set, or to determine how accurate a model might be, the largest data set possible should be used, and extremes of behaviour should be captured in addition to normal behaviour. It is insufficient to compare the simulations with data for only a few cycling regimes.

\section{Acknowledgements}

This work was supported by VITO and EnergyVille, Belgium. The research leading to these results was performed within the MAT4BAT project (http://mat4bat.eu/) and received funding from the European Union Seventh Framework Programme (FP7/2007-2013) under grant agreement no. 608931 and under the SolSThore project which receives the support of the European Union, the European Regional Development Fund ERDF, Flanders Innovation \& Entrepreneurship and the Province of Limburg. Financial support was also received from EVERLASTING project in the Horizon 2020 program of the European Union under the grant 'Electric Vehicle Enhanced Range, Lifetime And Safety Through INGenious battery management' (EVERLASTING-713771). DH acknowledges funding from the Faraday Institution.

\section{References}

\section{References}

[1] J. de Hoog, J.-M. Timmermans, D. Ioan-Stroe, M. Swierczynski, J. Jaguemont, S. Goutam, N. Omar, J. Van Mierlo, P. Van Den Bossche, Combined cycling and calendar capacity fade modeling of a NickelManganese-Cobalt Oxide Cell with real-life profile validation, Appl. Energ. 200 (2017) 47-61 (aug 2017).

[2] M. Petit, E. Prada, V. Sauvant-Moynot, Development of an empirical aging model for Li-ion batteries and application to assess the impact of Vehicle-to-Grid strategies on battery lifetime, Appl. Energ. 172 (2016) 398-407 (2016).

[3] J. M. Reniers, G. Mulder, S. Ober-Blöbaum, D. A. Howey, Improving optimal control of grid-connected lithium-ion batteries through more accurate battery and degradation modelling, J. Power Sources 379 (December 2017) (2018) 91-102 (mar 2018). 
[4] M. Schimpe, M. E. von Kuepach, M. Naumann, H. C. Hesse, K. Smith, A. Jossen, Comprehensive Modeling of Temperature-Dependent Degradation Mechanisms in Lithium Iron Phosphate Batteries, J. Electrochem. Soc. 165 (2) (2018) A181-A193 (jan 2018).

[5] K. Smith, A. Saxon, M. Keyser, B. Lundstrom, Ziwei Cao, A. Roc, Life prediction model for gridconnected Li-ion battery energy storage system, in: 2017 American Control Conference (ACC), IEEE, 2017, pp. 4062-4068 (may 2017).

[6] A. Barré, B. Deguilhem, S. Grolleau, M. Gérard, F. Suard, D. Riu, A review on lithium-ion battery ageing mechanisms and estimations for automotive applications, J. Power Sources 241 (2013) 680-689 (2013).

[7] A. Jokar, B. Rajabloo, M. Désilets, M. Lacroix, Review of simplified Pseudo-two-Dimensional models of lithium-ion batteries, J. Power Sources 327 (2016) 44-55 (sep 2016).

[8] D. U. Sauer, H. Wenzl, Comparison of different approaches for lifetime prediction of electrochemical systems-Using lead-acid batteries as example, J. Power Sources 176 (2) (2008) 534-546 (2008).

[9] C. Su, H. J. Chen, A review on prognostics approaches for remaining useful life of lithium-ion battery, IOP Conference Series: Earth and Environmental Science 93 (1) (2017).

[10] K. Goebel, B. Saha, A. Saxena, J. Celaya, J. Christophersen, Prognostics in Battery Health Management, IEEE Instru. Meas. Mag. 11 (4) (2008) 33-40 (aug 2008).

[11] A. A. Hussein, Capacity Fade Estimation in Electric Vehicle Li-Ion Batteries Using Artificial Neural Networks, IEEE Ind. Appl. 51 (3) (2015) 2321-2330 (may 2015).

[12] J. Snoek, H. Larochelle, R. P. Adams, Practical Bayesian Optimization of Machine Learning Algorithms, in: F. Pereira, C. J. C. Burges, L. Bottou, K. Q. Weinberger (Eds.), Advances in Neural Information Processing Systems 25, Curran Associates, Inc., 2012, pp. 2951-2959 (2012).

[13] A. Nuhic, T. Terzimehic, T. Soczka-Guth, M. Buchholz, K. Dietmayer, Health diagnosis and remaining useful life prognostics of lithium-ion batteries using data-driven methods, J. Power Sources 239 (2013) 680-688 (oct 2013).

[14] J. Zhang, J. Lee, A review on prognostics and health monitoring of Li-ion battery, J. Power Sources 196 (15) (2011) 6007-6014 (aug 2011).

[15] D. A. Howey, J. M. Reniers, SLIDE (2019). doi:10.5281/zenodo.2671696. URL https://github.com/davidhowey/SLIDE 
[16] W. A. Appiah, J. Park, S. Byun, M.-H. Ryou, Y. M. Lee, A Mathematical Model for Cyclic Aging of Spinel LiMn $<$ sub $>2</$ sub $>\mathrm{O}<$ sub $>4</$ sub $>/$ Graphite Lithium-Ion Cells, J. Electrochem. Soc. 163 (13) (2016) A2757-A2767 (2016).

[17] T. Ashwin, Y. M. Chung, J. Wang, Capacity fade modelling of lithium-ion battery under cyclic loading conditions, J. Power Sources 328 (2016) 586-598 (oct 2016).

[18] P. Barai, K. Smith, C.-F. Chen, G.-H. Kim, P. P. Mukherjee, Reduced Order Modeling of Mechanical Degradation Induced Performance Decay in Lithium-Ion Battery Porous Electrodes, J. Electrochem. Soc. 162 (9) (2015) A1751-A1771 (2015).

[19] J. Cannarella, C. B. Arnold, The Effects of Defects on Localized Plating in Lithium-Ion Batteries, J. Electrochem. Soc. 162 (7) (2015) A1365-A1373 (apr 2015).

[20] J. Christensen, J. Newman, Cyclable Lithium and Capacity Loss in Li-Ion Cells, J. Electrochem. Soc. 152 (4) (2005) A818 (2005).

[21] C. Delacourt, M. Safari, Life Simulation of a Graphite/LiFePO4 Cell under Cycling and Storage, J. Electrochem. Soc. 159 (8) (2012) A1283-A1291 (jul 2012).

[22] R. Deshpande, M. Verbrugge, Y.-T. Cheng, J. Wang, P. Liu, Battery Cycle Life Prediction with Coupled Chemical Degradation and Fatigue Mechanics, J. Electrochem. Soc. 159 (10) (2012) A1730-A1738 (2012).

[23] R. D. Deshpande, D. M. Bernardi, Modeling Solid-Electrolyte Interphase (SEI) Fracture: Coupled Mechanical/Chemical Degradation of the Lithium Ion Battery, J. Electrochem. Soc. 164 (2) (2017) A461-A474 (jan 2017).

[24] H. Ekstrom, G. Lindbergh, A Model for Predicting Capacity Fade due to SEI Formation in a Commercial Graphite/LiFePO4 Cell, J. Electrochem. Soc. 162 (6) (2015) A1003-A1007 (2015).

[25] H. Ge, T. Aoki, N. Ikeda, S. Suga, T. Isobe, Z. Li, Y. Tabuchi, J. Zhang, Investigating Lithium Plating in Lithium-Ion Batteries at Low Temperatures Using Electrochemical Model with NMR Assisted Parameterization, J. Electrochem. Soc. 164 (6) (2017) A1050-A1060 (2017).

[26] X. Jin, A. Vora, V. Hoshing, T. Saha, G. Shaver, R. E. García, O. Wasynczuk, S. Varigonda, Physicallybased reduced-order capacity loss model for graphite anodes in Li-ion battery cells, J. Power Sources 342 (2017) 750-761 (2017). 
[27] N. Kamyab, J. W. Weidner, R. E. White, Mixed Mode Growth Model for the Solid Electrolyte Interface (SEI), J. Electrochem. Soc. 166 (2) (2019) A334-A341 (jan 2019).

[28] F. M. Kindermann, J. Keil, A. Frank, A. Jossen, A SEI Modeling Approach Distinguishing between Capacity and Power Fade, J. Electrochem. Soc.164 (12) (2017) E287-E294 (aug 2017).

[29] C. Kupper, W. G. Bessler, Multi-Scale Thermo-Electrochemical Modeling of Performance and Aging of a LiFePO < sub $>4</$ sub $>$ /Graphite Lithium-Ion Cell, J. Electrochem. Soc. 164 (2) (2017) A304-A320 (2017).

[30] C. Kupper, B. Weißhar, S. Rißmann, W. G. Bessler, End-of-Life Prediction of a Lithium-Ion Battery Cell Based on Mechanistic Aging Models of the Graphite Electrode, J. Electrochem. Soc. 165 (14) (2018) A3468-A3480 (nov 2018).

[31] I. Laresgoiti, S. Käbitz, M. Ecker, D. U. Sauer, Modeling mechanical degradation in lithium ion batteries during cycling: Solid electrolyte interphase fracture, J. Power Sources 300 (2015) 112-122 (dec 2015).

[32] N. Legrand, B. Knosp, P. Desprez, F. Lapicque, S. Raël, Physical characterization of the charging process of a Li-ion battery and prediction of Li plating by electrochemical modelling, J. Power Sources 245 (2014) 208-216 (2014).

[33] D. Li, D. Danilov, Z. Zhang, H. Chen, Y. Yang, P. H. L. Notten, Modeling the SEI-Formation on Graphite Electrodes in LiFePO4 Batteries, J. Electrochem. Soc. 162 (6) (2015) A858-A869 (2015).

[34] X. Lin, J. Park, L. Liu, Y. Lee, A. M. Sastry, W. Lu, A Comprehensive Capacity Fade Model and Analysis for Li-Ion Batteries, J. Electrochem. Soc. 160 (10) (2013) A1701-A1710 (2013).

[35] R. Narayanrao, M. M. Joglekar, S. Inguva, A Phenomenological Degradation Model for Cyclic Aging of Lithium Ion Cell Materials, J. Electrochem. Soc. 160 (1) (2012) A125-A137 (nov 2012).

[36] G. Ning, B. N. Popov, Cycle Life Modeling of Lithium-Ion Batteries, J. Electrochem. Soc. 151 (10) (2004) A1584 (2004).

[37] M. B. Pinson, M. Z. Bazant, Theory of SEI Formation in Rechargeable Batteries: Capacity Fade, Accelerated Aging and Lifetime Prediction, J. Electrochem. Soc. 160 (2) (2012) A243-A250 (dec 2012).

[38] H. J. Ploehn, P. Ramadass, R. E. White, Solvent Diffusion Model for Aging of Lithium-Ion Battery Cells, J. Electrochem. Soc. 151 (3) (2004) A456 (2004). 
[39] J. Purewal, J. Wang, J. Graetz, S. Soukiazian, H. Tataria, M. W. Verbrugge, Degradation of lithium ion batteries employing graphite negatives and nickel-cobalt-manganese oxide + spinel manganese oxide positives: Part 2, chemical-mechanical degradation model, J. Power Sources 272 (2014) 1154-1161 (2014).

[40] P. Ramadass, B. Haran, P. M. Gomadam, R. White, B. N. Popov, Development of First Principles Capacity Fade Model for Li-Ion Cells, J. Electrochem. Soc. 151 (2) (2004) A196 (2004).

[41] A. V. Randall, R. D. Perkins, X. Zhang, G. L. Plett, Controls oriented reduced order modeling of solid-electrolyte interphase layer growth, J. Power Sources 209 (2012) 282-288 (2012).

[42] M. Safari, M. Morcrette, A. Teyssot, C. Delacourt, Multimodal Physics-Based Aging Model for Life Prediction of Li-Ion Batteries, J. Electrochem. Soc. 156 (3) (2009) A145 (2009).

[43] M. Safari, M. Morcrette, A. Teyssot, C. Delacourt, Life Prediction Methods for Lithium-Ion Batteries Derived from a Fatigue Approach, J. Electrochem. Soc. 157 (7) (2010) A892 (2010).

[44] M. Safari, C. Delacourt, Simulation-Based Analysis of Aging Phenomena in a Commercial Graphite/LiFePO4 Cell, J. Electrochem. Soc. 158 (12) (2011) A1436 (2011).

[45] F. Single, B. Horstmann, A. Latz, Revealing SEI Morphology: In-Depth Analysis of a Modeling Approach, J. Electrochem. Soc. 164 (11) (2017) E3132-E3145 (2017).

[46] A. A. Tahmasbi, T. Kadyk, M. H. Eikerling, Statistical Physics-Based Model of Solid Electrolyte Interphase Growth in Lithium Ion Batteries, J. Electrochem. Soc. 164 (6) (2017) A1307-A1313 (2017).

[47] M. Tang, S. Lu, J. Newman, Experimental and Theoretical Investigation of Solid-Electrolyte-Interphase Formation Mechanisms on Glassy Carbon, J. Electrochem. Soc. 159 (11) (2012) A1775-A1785 (2012).

[48] X.-G. Yang, Y. Leng, G. Zhang, S. Ge, C.-Y. Wang, Modeling of lithium plating induced aging of lithium-ion batteries: Transition from linear to nonlinear aging, J. Power Sources 360 (2017) 28-40 (aug 2017).

[49] S. J. Harris, P. Lu, Effects of inhomogeneities -Nanoscale to mesoscale -on the durability of Li-ion batteries, J. Phys. Chem. C 117 (13) (2013) 6481-6492 (2013).

[50] S. J. Harris, D. J. Harris, C. Li, Failure statistics for commercial lithium ion batteries: A study of 24 pouch cells, J. Power Sources 342 (2017) 589-597 (2017). 
[51] T. C. Bach, S. F. Schuster, E. Fleder, J. Müller, M. J. Brand, H. Lorrmann, A. Jossen, G. Sextl, Nonlinear aging of cylindrical lithium-ion cells linked to heterogeneous compression, J. Energy Storage 5 (2016) 212-223 (2016).

[52] M. Guo, G. Sikha, R. E. White, Single-Particle Model for a Lithium-Ion Cell: Thermal Behavior, J. Electrochem. Soc. 158 (2) (2011) A122 (2011).

[53] P. Verma, P. Maire, P. Novák, A review of the features and analyses of the solid electrolyte interphase in Li-ion batteries, Electrochim. Acta 55 (22) (2010) 6332-6341 (sep 2010).

[54] V. A. Agubra, J. W. Fergus, The formation and stability of the solid electrolyte interface on the graphite anode, J. Power Sources 268 (2014) 153-162 (dec 2014).

[55] M. Gauthier, T. J. Carney, A. Grimaud, L. Giordano, N. Pour, H.-H. Chang, D. P. Fenning, S. F. Lux, O. Paschos, C. Bauer, F. Maglia, S. Lupart, P. Lamp, Y. Shao-Horn, Electrode-Electrolyte Interface in Li-Ion Batteries: Current Understanding and New Insights, The J. Phys. Chem. Lett. 6 (22) (2015) 4653-4672 (nov 2015).

[56] P. Lu, C. Li, E. W. Schneider, S. J. Harris, Chemistry, impedance, and morphology evolution in solid electrolyte interphase films during formation in lithium ion batteries, J. Phys. Chem. C 118 (2) (2014) 896-903 (2014).

[57] Z. Zhuo, P. Lu, C. Delacourt, R. Qiao, K. Xu, F. Pan, S. J. Harris, W. Yang, Breathing and oscillating growth of solid-electrolyte-interphase upon electrochemical cycling, Chem. Commun. 54 (7) (2018) 814817 (2018).

[58] Basquin, The exponential law of endurance tests, in: Proc. ASTM 10, 1910, pp. 625-630 (1910).

[59] Y. Dai, L. Cai, R. E. White, Simulation and analysis of stress in a Li-ion battery with a blended LiMn 2 O 4 and LiNi 0.8 Co 0.15 Al 0.05 O 2 cathode, J. Power Sources 247 (2014) 365-376 (feb 2014).

[60] B. Wu, W. Lu, A battery model that fully couples mechanics and electrochemistry at both particle and electrode levels by incorporation of particle interaction, J. Power Sources 360 (2017) 360-372 (aug 2017).

[61] E. Bohn, T. Eckl, M. Kamlah, R. McMeeking, A Model for Lithium Diffusion and Stress Generation in an Intercalation Storage Particle with Phase Change, J. Electrochem. Soc. 160 (10) (2013) A1638-A1652 (jul 2013). 
[62] J. Li, N. Lotfi, R. G. Landers, J. Park, A Single Particle Model for Lithium-Ion Batteries with Electrolyte and Stress-Enhanced Diffusion Physics, J. Electrochem. Soc. 164 (4) (2017) A874-A883 (feb 2017).

[63] R. Fu, M. Xiao, S. Y. Choe, Modeling, validation and analysis of mechanical stress generation and dimension changes of a pouch type high power Li-ion battery, J. Power Sources 224 (2013) 211-224 (2013).

[64] Q. Liu, C. Du, B. Shen, P. Zuo, X. Cheng, Y. Ma, G. Yin, Y. Gao, Understanding undesirable anode lithium plating issues in lithium-ion batteries, RSC Adv. 6 (91) (2016) 88683-88700 (2016).

[65] J. Vetter, P. Novák, M. Wagner, C. Veit, K.-C. Möller, J. Besenhard, M. Winter, M. Wohlfahrt-Mehrens, C. Vogler, A. Hammouche, Ageing mechanisms in lithium-ion batteries, J. Power Sources 147 (1-2) (2005) 269-281 (sep 2005).

[66] A. Bizeray, S. Zhao, S. Duncan, D. Howey, Lithium-ion battery thermal-electrochemical model-based state estimation using orthogonal collocation and a modified extended Kalman filter, J. Power Sources 296 (2015) 400-412 (nov 2015).

[67] S. Pelletier, O. Jabali, G. Laporte, M. Veneroni, Battery degradation and behaviour for electric vehicles: Review and numerical analyses of several models, Transportation Research Part B: Methodological 103 (2017) 158-187 (sep 2017).

[68] European Commission, EVERLASTING (2018). URL https://cordis.europa.eu/project/rcn/204971/factsheet/en https://ec.europa.eu/inea/en/horizon-2020/projects/h2020-transport/green-vehicles/everlasting https://everlasting-project.eu/

[69] LG Chem, Rechargeable Lithium Ion Battery Model : INR18650 MJ1 3500mAh, PRODUCT SPECIFICATION (jan 2014).

URL https://www.nkon.nl/sk/k/Specification INR18650MJ122.08.2014.pdf

[70] European Commission, MAT4BAT (2016).

URL http://cordis.europa.eu/project/rcn/109052_en.html

[71] Kokam, KOKAM Li-ion / Polymer Cell (2016).

URL http://kokam.com/wp-content/uploads/2016/03/SLPB-Cell-Brochure.pdf

[72] M. Dubarry, A. Devie, K. McKenzie, Durability and reliability of electric vehicle batteries under electric utility grid operations: Bidirectional charging impact analysis, J. Power Sources 358 (2017) 39-49 (aug 2017). 
[73] J. Schmalstieg, S. Käbitz, M. Ecker, D. U. Sauer, A holistic aging model for Li(NiMnCo)O2 based 18650 lithium-ion batteries, J. Power Sources 257 (2014) 325-334 (jul 2014).

[74] K. Jalkanen, J. Karppinen, L. Skogström, T. Laurila, M. Nisula, K. Vuorilehto, Cycle aging of commercial NMC/graphite pouch cells at different temperatures, Appl. Energ. 154 (2015) 160-172 (sep 2015).

[75] Y. Wu, P. Keil, S. F. Schuster, A. Jossen, Impact of Temperature and Discharge Rate on the Aging of a LiCoO 2 /LiNi 0.8 Co 0.15 Al 0.05 O 2 Lithium-Ion Pouch Cell, J. Electrochem. Soc. 164 (7) (2017) A1438-A1445 (may 2017).

[76] M. Klett, R. Eriksson, J. Groot, P. Svens, K. Ciosek Högström, R. W. Lindström, H. Berg, T. Gustafson, G. Lindbergh, K. Edström, Non-uniform aging of cycled commercial LiFePO4//graphite cylindrical cells revealed by post-mortem analysis, J. Power Sources 257 (2014) 126-137 (jul 2014).

[77] S. F. Schuster, T. Bach, E. Fleder, J. Müller, M. Brand, G. Sextl, A. Jossen, Nonlinear aging characteristics of lithium-ion cells under different operational conditions, J. Energy Storage 1 (1) (2015) 44-53 (jun 2015). 